\title{
Frequency Spectrum Regularization for Pattern Noise Removal Based on Image Decomposition
}

\author{
Keiichiro Shirai \\ Shinshu Univ.
}

\author{
Shunsuke Ono \\ Tokyo Institute of Tech.
}

\author{
Masahiro Okuda \\ Univ. of Kitakyushu
}

\begin{abstract}
This paper deals with a mixed norm of complex vectors, i.e., the sum of amplitude spectra, and its minimization problem. A combination of this mixed norm and image decomposition problem works well for reduction and decomposition of pattern noise that arises when scanning old photographs with granulated surface. Generally, the spectral distribution of natural images decreases smoothly from low frequency band toward high frequency band, while that of pattern noise is distributed sparsely. Therefore, we assume that an observed image consists of a latent image component and a pattern noise component, and characterize them by using the total variation function and the proposed function, respectively. This enables a reasonable decomposition of the two components. Compared to similar decomposition methods such as Robust PCA, our method has a good decomposition accuracy for this task, and low computational cost.
\end{abstract}

\section{INTRODUCTION}

When scanning old photographic paper as digital data, noisy patterns sometime appear on the images as shown in Fig. 1(a) due to the light of the scanner reflected from the rough granulated surface of the paper, and one can see particulate texture appearing over the whole region of the image. To reduce this kind of artifact physically, changing a scanner device or shooting the picture under adjusted light positions is effective, but those methods are actually time-consuming. Therefore, we aim at the software-based reduction of pattern noise, and propose an equation model for separating the original image and the pattern noise.

Our separation model and the algorithm are based on robust principal component analysis (RPCA) proposed by Wright et al. [1] and reflection component removal proposed by $\mathrm{Li}$ et al. [2]. These methods assume that an observed image consists of two components, and separate it into an ideal image component (hereafter called "latent" image) and an artifact image component different from shot noise. When expressing the model as a mathematical optimization problem, in addition to the data fidelity expressing the observation process, these methods utilize some regularizations expressing the feature of each component, and separate image components by utilizing the differences of the regularizations.

In the above methods, it is important how to characterize the artifact image. The RPCA [1], [3] regards luminance change (specular reflection) caused by reflected light as an artifact component, and characterize it so as to minimize the sum of absolute luminance values over the whole image (i.e., $\ell_{1}$ norm). The reflection component removal [2] deals with a faint and blurred reflection image component, and characterize it so
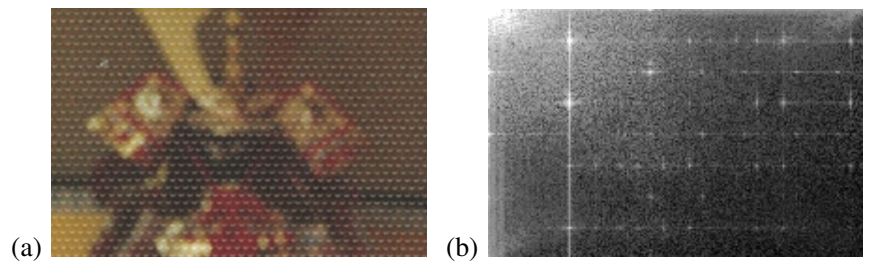

Fig. 1. Pattern noise arises over a scanned image (a) and artifact pattern arises over the spectral component (b). In the spectral image, the 1st quadrant is shown and the intensity range is normalized for displaying the detail. The top left and the bottom right part correspond to the low and the high frequency band.

as to minimize the sum of luminance variations over the whole image (Tikhonov regularization).

In this paper, we regard pattern noise as an artifact component. As shown in Fig. 1(b), in the spectra of an image in which the same artifact pattern arises iteratively, spectra corresponding to the pattern tend to appear sparsely together with high peak values. Therefore, we characterize it so as to minimize the sum of amplitude spectra (hereafter called "spectra" for simplicity) over the whole images frequencies.

Using the aforementioned component separation model and the vector norm of complex numbers, we propose an equation model for separating a latent image and a pattern noise image. In the model, the latent image is characterized by total variation minimization, while the pattern noise is characterized by $\ell_{1}$ norm minimization of spectral values with a zero mean constraint. This model can be expressed as a convex optimization problem, so the global solution is given by using appropriate algorithms. Additionally, subproblems appearing in the iterative algorithm are calculated efficiently.

\section{MIXED NORMS AND PROXIMITY OPERATORS FOR REAL AND COMPLEX VECTORS}

We first describe norm functions and proximity operators for real and complex vectors, which are used in the proposal method. Since definitions for the real-valued vector are well known, the explanation is mainly intended for complex-valued vectors.

Mixed $\ell_{2,1}$ norm of real vectors and its proximity operator: At a pixel $i$, when handling $K$ multiple values $\left\{x_{i, j}\right\}_{j=1}^{K}$ (hereafter called "group variables"), and considering the sparsity of the group variables, mixed norms [4], [5] are usually used. The mixed $\ell_{p, q}$ norm of a vector consisting of all group variables of $N$ pixels $\mathbf{x}:=$ $\left[x_{1,1}, x_{1,2}, \ldots, x_{2,1}, x_{2,2}, \ldots, x_{N, K}\right]^{\top} \in \mathbb{R}^{K N}$ is given by 
$\|\mathbf{x}\|_{p, q}:=\left(\sum_{i}\left(\sum_{j}\left|x_{i, j}\right|^{p}\right)^{q / p}\right)^{1 / q}$. When $p=q$, it becomes the standard $\ell_{p}$ norm. In this paper, the $\ell_{2,1}$ norm is used:

$$
\|\mathbf{x}\|_{2,1}:=\sum_{i} \sqrt{\sum_{j}\left|x_{i, j}\right|^{2}}
$$

This $\ell_{2,1}$ norm function is a convex function giving the minimum value at the origin where all values become $x_{i, j}=0$. Although it is non-differentiable at the origin, the function is proximable, i.e., its proximity operator described in the next paragraph is easily obtained.

Then, we describe the proximity operator corresponding to the $\ell_{2,1}$ norm. The operator is used as an alternative of gradient calculation for non-differentiable convex functions $F$, and defined as a map: $\operatorname{prox}_{\gamma F}: \mathbb{R}^{N} \rightarrow \mathbb{R}^{N}: \mathbf{x} \mapsto$ $\arg \min _{\mathbf{x}^{\prime}} \gamma F\left(\mathbf{x}^{\prime}\right)+\frac{1}{2}\left\|\mathbf{x}^{\prime}-\mathbf{x}\right\|_{2}^{2}$. The calculation corresponding to the proximity operator of the $\ell_{2,1}$ norm, i.e., $\operatorname{prox}_{\gamma\|\cdot\|_{2,1}}(\mathbf{x})$, is known to be given as follows [6]:

$$
\forall_{i} x_{i, j}^{\prime}:=s_{i} x_{i, j}, \quad s_{i}:=1-\frac{\gamma}{\max \left(\gamma, \sqrt{\sum_{j}\left|x_{i, j}\right|^{2}}\right)},
$$

where $s_{i}$ is the scaling variable, and the function $\max (\cdot, \cdot)$ returns the maximum variable. In this calculation, firstly, the $\ell_{2}$ norm of group variables at each pixel is computed. If the value is sufficiently small lower than $\gamma$, all the group variables are erased $\left(s_{i}=0\right.$, so $\left.x_{i, j}^{\prime}=0\right)$. Otherwise, the group variables are scaled related to the magnitude of the norm.

Mixed $\ell_{C, 1}$ norm of complex vectors and its proximity operator: At a pixel $i$, when handling a complex number as $c_{i}:=c_{i}^{\mathcal{R}}+c_{i}^{\mathcal{I}} j \in \mathbb{C}^{1}$, the norm is usually defined as $|\cdot|_{C}: \mathbb{C}^{1} \rightarrow \mathbb{R}^{1}: c_{i} \mapsto \sqrt{\left(c_{i}^{\mathcal{R}}\right)^{2}+\left(c_{i}^{\mathcal{I}}\right)^{2}}$, where $C_{C}$ is added to distinguish it from the real-valued vector norm.

Then, the mixed $\ell_{C, 1}$ norm of a complex vector consisting of all complex elements of $N$ pixels $\mathbf{c}=\left[c_{1}, \ldots, c_{N}\right] \in \mathbb{C}^{N}$ (i.e., the mixed norm of the complex-valued norm and the $\ell_{1}$ norm, generally called the " $\ell_{1}$ norm of complex vectors") is given by

$$
\|\cdot\|_{C, 1}: \mathbb{C}^{N} \rightarrow \mathbb{R}^{1}: \mathbf{c} \mapsto \sum_{i}\left|c_{i}\right|_{C}
$$

This $\ell_{C, 1}$ norm function is a convex function on the complex plane and gives the minimum value at the origin where all values become $\left(c_{i}^{\mathcal{R}}, c_{i}^{\mathcal{I}}\right)=(0,0)$.

The proximity operator corresponding to the $\ell_{C, 1}$ norm is defined as $\operatorname{prox}_{\gamma\|\cdot\|_{C, 1}}(\mathbf{c}):=\min _{\mathbf{c}^{\prime}} \sum_{i} \gamma\left|c_{i}\right|_{C}+\frac{1}{2}\left|c^{\prime}{ }_{i}-c_{i}\right|_{C}^{2}$. This function is non-differentiable at the origin, but otherwise differentiable, so we can derive the following calculation by organizing conditions giving the minimum value:

$$
\left\{\begin{array}{l}
c^{\prime \mathcal{R}}:=s \cdot c_{i}^{R} \\
c_{i}^{\prime \mathcal{I}}:=s \cdot c_{i}^{I}
\end{array} \quad \text { where } \quad s:=\left(1-\frac{\gamma}{\max \left(\gamma,\left|c_{i}\right|_{C}\right)}\right) .\right.
$$

This is the same calculation as the mixed $\ell_{2,1}$ norm shown in (2). As for the complex vector, basically, we can regard the real number and the imaginary number as a group variables [3].

\section{PROpOSED METHOD}

This section describes a formulation as a convex optimization problem for separating a degraded image as shown in Fig. 1(a) into a latent image and a pattern image. We first describe the image observation model (data fidelity term), and then describe characterization methods (regularization terms) for separating an image into each component.

Construction model of image components and the data fidelity term: We treat an observed image as a grayscale image of $N$ pixels, and as a column vector $\mathbf{y} \in \mathbb{R}^{N}$ consisting of pixel values. Although Fig. 1(a) is a RGB color image, in this paper for simplicity, our method processes each color channel independently, and does not consider the relationship of colors.

Then, we define the latent image $l \in \mathbb{R}^{N}$ and the pattern noise image $\mathbf{e} \in \mathbb{R}^{N}$, and define the relationship between them and the observed image as $\mathbf{y}:=\mathbf{l}+\mathbf{e}^{1}$. Additionally, we make the following assumptions: the mean value of the pattern noise is assumed as 0; shot noise arising in shooting is sufficiently small and omitted from the model; the noise is additive white Gaussian noise.

When handling the above observation model as an optimization problem, the model is generally expressed as a minimization problem of the $\ell_{2}$ norm of observation errors. On the other hand, our method formulates it as a $\ell_{2}$ ball constraint for tractability: $\|\mathbf{y}-(\mathbf{l}+\mathbf{e})\|_{2} \leq \eta, \mathbf{1}^{\top} \mathbf{e}=0$, where $\eta(\geq 0)$ is a user defined tolerance. $\mathbf{1} \in 1^{N}$ is a vector of ones, and this constraint makes the mean value (sum) be 0 .

Characterization for the latent image: TV regularization: In many cases, luminance values of an image are constant and flat in many regions, and the luminance values are sharply changed around object edges. In the case of clear images, the sum of luminance variations (total variation: TV) becomes small [7]. We also consider this TV minimization, and use the mixed $\ell_{2,1}$ norm and a differential filter, define the character as $\|\mathbf{D l}\|_{2,1}$, where $\mathbf{D}:=\left[\mathbf{D}_{v}^{\top}, \mathbf{D}_{h}^{\top}\right]^{\top} \in \mathbb{R}^{2 N \times N}$ is the differential filter matrix consisting of the horizontal and vertical differential filters $\mathbf{D}_{v}$ and $\mathbf{D}_{h} \in \mathbb{R}^{N \times N}$. Note that the differential filter is a general one having 2-tap coefficients $[-1,1]$, but is a circular type filter in order to simplify the calculation described later. Additionally, the transposed filter matrix $\mathbf{D}^{\top}$ corresponds to the filter having $180^{\circ}$ rotated coefficients $[1,-1]$.

Characterization for the pattern noise: spectral regularization: In a pattern image in which the same artifact pattern arises iteratively, its spectra over frequency coordinates corresponding to the pattern tend to become large. When the same pattern appears strongly, the sum of spectral values (total spectra) becomes small. We focus on this character, and uses the mixed $\ell_{C, 1}$ norm and the Fourier transform, define the minimization of spectra as $\|\mathbf{F e}\|_{C, 1}$, where $\mathbf{F} \in \mathbb{R}^{N \times N}$ denotes the fast discrete Fourier transform. Note that we define

\footnotetext{
${ }^{1}$ When assuming that pattern noise is caused by reflected light, a pixel-wise multiplicative model $\mathbf{y}:=\mathbf{l} \circ \mathbf{e}$ is appropriate. However, we only consider the basic additive model due to the page limitation.
} 
$\mathbf{F}$ as a unitary matrix so that the sum of the square of a function is equal to the sum of the square of its transform 2 . In this case, the complex conjugate transpose (Hermitian transpose) $\mathbf{F}^{H}$ and the inverse Fourier transform $\mathbf{F}^{-1}$ become equivalent $\mathbf{F}^{H}=\mathbf{F}^{-1}$, which makes handling the following equations be easier.

Formulation for pattern noise decomposition: Combining the aforementioned data fidelity term, regularization terms, and constraint, we propose the formulation for noise pattern separation as

$$
\begin{aligned}
& \min _{\mathbf{l}, \mathbf{e}}\|\mathbf{D} \mathbf{l}\|_{2,1}+\lambda\|\mathbf{F e}\|_{C, 1}, \\
& \text { s.t. }\|\mathbf{y}-(\mathbf{l}+\mathbf{e})\|_{2} \leq \eta, \quad \mathbf{1}^{\top} \mathbf{e}=0,
\end{aligned}
$$

where the parameter $\lambda$ in the objective function balances the 1 st and 2 nd terms. The parameter values of $\lambda$ and the tolerance parameter $\eta$ are described in the experimental results $\mathrm{V}$. Note that we can embed the constraint $\mathbf{1}^{\top} \mathbf{e}=0$ into the minimization of spectra, and handle it with just a few modifications in the algorithm described later, so omit it for simplicity.

\section{Solution Algorithm Using ADMM}

For solving the proposed equation in (5), we can use some algorithms, and here show an algorithm using ADMM (alternating direction method of multiplies) [8], [9]. Due to the page limitation, we omit how to derivate the algorithm and calculations in each step.

First, we make preparations for adding the constraints to the object function by using the method of Lagrange multipliers. To express the $\ell_{2}$ ball constraint $\mathcal{B}_{\mathbf{y}, \eta}:=\left\{\mathbf{x} \mid\|\mathbf{x}-\mathbf{y}\|_{2} \leq\right.$ $\eta\}$ as a regularizer, we introduce an indicator function $\iota_{\mathcal{B}_{\mathbf{y}}, \eta}$ : $\min _{\mathbf{l}, \mathbf{e}}\|\mathbf{D} \mathbf{l}\|_{2,1}+\lambda\|\mathbf{F e}\|_{C, 1}+\iota_{\mathcal{B}_{\mathbf{y}, \eta}}(\mathbf{l}+\mathbf{e})$. The indicator function is defined as $\iota_{\mathcal{C}}(\mathbf{x}):=\left\{\begin{array}{ll}0 & \mathbf{x} \in \mathcal{C}, \\ \infty & \text { otherwise, }\end{array}\right.$ where the set $\mathcal{C}$ is a convex set. In the case of the $\ell_{2}$ ball, the indicator function becomes a convex function whose proximity operator can be easily calculated (see (10)). Then, these functions are nondifferentiable convex functions, so we replace the variables to make the functions more tractable form, $\mathbf{z}_{l}:=\mathbf{D l}, \mathbf{z}_{e}:=\mathbf{F e}$, $\mathbf{z}:=\mathbf{l}+\mathbf{e}$, and add these equality constraints to the objective function as

$$
\begin{aligned}
& L(\mathbf{l}, \mathbf{e},\{\mathbf{z}\},\{\mathbf{u}\}):=\left\|\mathbf{z}_{l}\right\|_{2,1}+(\rho / 2)\left\|\mathbf{z}_{l}-\left(\mathbf{D l}+\mathbf{u}_{l}\right)\right\|_{2}^{2} \\
& +\lambda\left\|\mathbf{z}_{e}\right\|_{C, 1}+(\rho / 2)\left\|\mathbf{z}_{e}-\left(\mathbf{F e}+\mathbf{u}_{e}\right)\right\|_{2}^{2} \\
& +\iota_{\mathcal{B}_{\mathbf{y}, \eta}}(\mathbf{z})+(\rho / 2)\|\mathbf{z}-(\mathbf{l}+\mathbf{e}+\mathbf{u})\|_{2}^{2},
\end{aligned}
$$

where $\mathbf{u}_{l}, \mathbf{u}_{e}, \mathbf{u}$ are Lagrange multipliers. $\rho$ is the step size to control the convergence of the ADMM, and set to $\rho=1$ in this paper.

Algorithm of ADMM: When solving (6), the following minimization subproblems w.r.t. each variable are solved itera-

\footnotetext{
${ }^{2}$ The Fourier transform function used in software is generally non-unitary, and the output is scaled as $\sqrt{N} \mathbf{F x}$. When using these non-unitary transformation, we need multiply $\lambda$ by $\sqrt{N}$ described later in Sec. 5 .
}

tively, and each variable is solved while fixing other variables. The calculation at $t$-th iteration is given by

$$
\mid \begin{aligned}
& \mathbf{l}^{t+1}, \mathbf{e}^{t+1}:=\min _{\mathbf{x}} L\left(\mathbf{z}_{l}^{t}, \mathbf{z}_{e}^{t}, \mathbf{z}^{t}, \mathbf{u}_{l}^{t}, \mathbf{u}_{e}^{t}, \mathbf{u}^{t}\right), \\
& \mathbf{z}_{l}^{t+1}:=\min _{\mathbf{z}_{l}} L\left(\mathbf{x}^{t+1}, \mathbf{u}_{l}^{t}\right)=\operatorname{prox}_{\frac{1}{\rho}\|\cdot\|_{2,1}}\left(\mathbf{D l}^{t+1}+\mathbf{u}_{l}^{t}\right), \\
& \mathbf{z}_{e}^{t+1}:=\min _{\mathbf{z}_{e}} L\left(\mathbf{x}^{t+1}, \mathbf{u}_{e}^{t}\right)=\operatorname{prox}_{\frac{\lambda}{\rho}\|\cdot\|_{C, 1}}\left(\mathbf{F e}^{t+1}+\mathbf{u}_{e}^{t}\right), \\
& \mathbf{z}^{t+1}:=\min _{\mathbf{z}} L\left(\mathbf{x}^{t+1}, \mathbf{u}^{t}\right)=\operatorname{prox}_{\frac{1}{\rho}} \iota_{\mathcal{B}_{\mathbf{y}, \eta}}\left(\mathbf{l}^{t+1}+\mathbf{e}^{t+1}+\mathbf{u}^{t}\right), \\
& \mathbf{u}_{l}^{t+1}:=\min _{\mathbf{u}_{l}} L\left(\mathbf{x}^{t+1}, \mathbf{z}_{l}^{t+1}\right)=\mathbf{u}_{l}^{t}+\mathbf{D l}^{t+1}-\mathbf{z}_{l}^{t+1}, \\
& \mathbf{u}_{e}^{t+1}:=\min _{\mathbf{u}_{e}} L\left(\mathbf{x}^{t+1}, \mathbf{z}_{e}^{t+1}\right)=\mathbf{u}_{e}^{t}+\mathbf{F e}^{t+1}-\mathbf{z}_{e}^{t+1} \\
& \mathbf{u}^{t+1}:=\min _{\mathbf{u}} L\left(\mathbf{x}^{t+1}, \mathbf{z}^{t+1}\right)=\mathbf{u}^{t}+\mathbf{l}^{t+1}+\mathbf{e}^{t+1}-\mathbf{z}^{t+1}
\end{aligned}
$$

The details how to solve $\mathbf{l}$, e, and the proximity operators are described later. The number of iterations is shown in Sec. V. As for the initial values, we set $\mathbf{l}^{t=0}=\mathbf{y}$, and other variables to zero vectors.

Solutions of 1 and e: In (6), all terms related to 1 and e are $\ell_{2}$ norms. The minimum cost is given when the gradients of $\mathbf{l}$ and $\mathbf{e}$ become $\mathbf{0}$. Calculating $\frac{\partial}{\partial \mathbf{l}} L=\mathbf{0}$ and $\frac{\partial}{\partial \mathbf{e}} L=\mathbf{0}$, we get the following equation in the form $\left(\begin{array}{ll}\mathbf{A}_{11} & \mathbf{A}_{12} \\ \mathbf{A}_{21} & \mathbf{A}_{22}\end{array}\right)\left(\begin{array}{l}\mathbf{x}_{1} \\ \mathbf{x}_{2}\end{array}\right)=\left(\begin{array}{l}\mathbf{b}_{1} \\ \mathbf{b}_{2}\end{array}\right)$ as

$$
\left(\begin{array}{cc}
\mathbf{I}+\mathbf{D}^{\top} \mathbf{D} & \mathbf{I} \\
\mathbf{I} & \mathbf{I}+\mathbf{F}^{H} \mathbf{F}
\end{array}\right)\left(\begin{array}{l}
\mathbf{l} \\
\mathbf{e}
\end{array}\right)=\left(\begin{array}{c}
\left(\mathbf{z}^{t}-\mathbf{u}^{t}\right)+\mathbf{D}^{\top}\left(\mathbf{z}_{l}^{t}-\mathbf{u}_{l}^{t}\right) \\
\left(\mathbf{z}^{t}-\mathbf{u}^{t}\right)+\mathbf{F}^{H}\left(\mathbf{z}_{e}^{t}-\mathbf{u}_{e}^{t}\right)
\end{array}\right),
$$

where $\mathbf{F}^{H} \mathbf{F}=\mathbf{I}$ can be simplified. When the differential filter is defined as circular convolution, each block $\mathbf{A}_{i j}$ becomes a special matrix having a BCCB (block circulant circulant block) structure, and it can be diagonalized by Fourier transformation as $\mathbf{F} \mathbf{A}_{i j} \mathbf{F}^{-1}$. The inverse of a matrix consisting of diagonalized blocks is easily obtained [10].

We define a function computing the transfer function of a filter corresponding to each block as $\mathscr{F}\left(\mathbf{A}_{i j}\right):=$ $\operatorname{diag}\left(\mathbf{F} \mathbf{A}_{i j} \mathbf{F}^{-1}\right)$, where $\operatorname{diag}(\cdot)$ extracts diagonal values of the matrix as a vector. Additionally, we define Fourier transform and inverse Fourier transform as $\mathscr{F}\left(\mathbf{b}_{i}\right):=\mathbf{F b}_{i}$ and $\mathscr{F}^{-1}\left(\mathbf{b}_{i}\right):=\mathbf{F}^{-1} \mathbf{b}_{i}$. Using these functions, the solution is obtained as

$$
\begin{aligned}
\mathbf{l}^{t+1} & =\mathscr{F}^{-1}\left(\frac{\mathscr{F}\left(\mathbf{A}_{22}\right) \circ \mathscr{F}\left(\mathbf{b}_{1}\right)-\mathscr{F}\left(\mathbf{A}_{12}\right) \circ \mathscr{F}\left(\mathbf{b}_{2}\right)}{\mathscr{F}(\boldsymbol{\Delta})}\right), \\
\mathbf{e}^{t+1} & =\mathscr{F}^{-1}\left(\frac{\mathscr{F}\left(\mathbf{A}_{11}\right) \circ \mathscr{F}\left(\mathbf{b}_{2}\right)-\mathscr{F}\left(\mathbf{A}_{21}\right) \circ \mathscr{F}\left(\mathbf{b}_{1}\right)}{\mathscr{F}(\boldsymbol{\Delta})}\right),
\end{aligned}
$$

where $\circ$ and fraction denote element-wise multiplication and division. The denominator is given by $\mathscr{F}(\boldsymbol{\Delta})=\mathscr{F}\left(\mathbf{A}_{11}\right) \circ$ $\mathscr{F}\left(\mathbf{A}_{22}\right)-\mathscr{F}\left(\mathbf{A}_{12}\right) \circ \mathscr{F}\left(\mathbf{A}_{21}\right)$.

Solutions of $\mathrm{z}_{l}, \mathrm{z}_{e}$, and $\mathrm{z}$ : calculation of proximity operators: As for proximity operators corresponding to the mixed norms $\operatorname{prox}_{\frac{1}{\rho}\|\cdot\|_{2,1}}(\cdot)$ and $\operatorname{prox}_{\frac{\lambda}{\rho}\|\cdot\|_{C, 1}}(\cdot)$, they can be calculated as described in (2) and (4). Then, as for the constraint that makes the mean value of $\mathbf{e}$ be 0 , it can be realized by set the direct current (DC) component of $\mathbf{z}^{t+1}$ to 0 : $\left(\mathbf{z}_{l}^{t+1}\right)_{\mathrm{DC}}:=0$. Finally, the proximity operator corresponding to the $\ell_{2}$ ball is calculated as

$$
\operatorname{prox}_{\gamma} \iota_{\mathcal{B}_{\mathbf{y}, \eta}}(\mathbf{v}):=\left\{\begin{array}{cl}
\mathbf{v} & \|\mathbf{v}-\mathbf{y}\|_{2} \leq \eta, \\
\mathbf{y}+\eta \frac{\mathbf{v}-\mathbf{y}}{\|\mathbf{v}-\mathbf{y}\|_{2}} & \text { otherwise. }
\end{array}\right.
$$




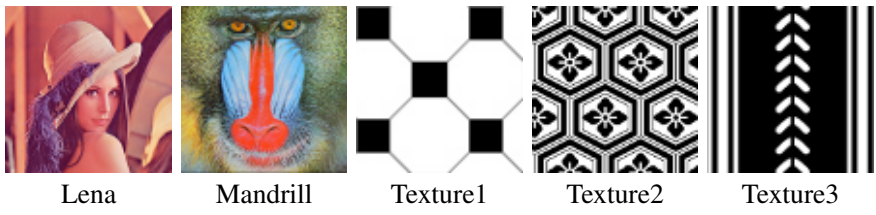

Fig. 2. Original latent images and pattern images used in the simulation experiment.

\section{EXPERIMENTAL RESULTS}

This section shows, firstly, simulation results using the original latent and pattern images, and then actual results using scanned and shot images. All parameters used in this experiment are fixed: as for our model, $\lambda=3$ and $\eta=0$; as for the ADMM, $\rho=1$ and the number of iteration is 100 .

Simulation experiment: Preparing original latent and pattern images as shown in Fig. 2, we generate observation images by linearly mixed them, and then separating the observation images into latent and pattern images again, finally compare PSNRs (peak signal and noise ratio) between the original images and resultant images. We used the "Lena" and "Mandrill" as the latent images, while free resources ${ }^{3}$ as the pattern images. The image sizes are all $512 \times 512$ and its luminance is normalized within the range $[0,1]$. As for the pattern images, partial $86 \times 86$ regions are shown for displaying the details. Note that, in generating observation images, the luminance values of each pattern image are centered so that the mean value to be 0 , and then scaled to half $(\times 0.5)$, finally added to the latent images.

Qualitative evaluation of resultant images: Figure 3, additionally Fig. 4(d), show a few of resultant images. In Fig. 3, from the left, (a) original latent image, (b) original pattern image (the gray color corresponds to the zero luminance), (c) mixed image, (d) obtained latent image, and (e) obtained pattern image are shown. The bottom row shows spectral images of them. Here, in Fig. 4(d), we used an easier pattern to be separated, which has continuous circularity at the boundary of the image and has isotropic pattern with a similar texture in horizontal and vertical directions. In contrast, we used a difficult pattern in Fig. 3, which is anisotropic texture and has non-continuous circularity, especially in the horizontal direction.

In the easier pattern in Fig. 4(d), although the pattern is faintly left in the latent image, one can see that the separation gives a good result. On the other hand, in the difficult pattern in Fig. 3(d) and (e), the pattern is left in the horizontal direction toward which the pattern has non-continuous circularity. In its spectra, line-like textures included in (c) can be removed (d). However, high frequency peaks (corresponding to the fur of the Mandrill) results in being wrongly separated.

Comparison with related methods: Figure 4 shows comparison results with the aforementioned RPCA [1] and reflection removal [2]. The parameters of each method are adjusted to be able to extract the pattern components. Here, we blur the

\footnotetext{
${ }^{3}$ WArgo: http://japanese-pattern.info/
}

TABLE I

PSNRS $(\mathrm{dB})$ between decomposed images and original images in the simulation experiment.

with continuous texture loop

\begin{tabular}{|c|c|c|c|c|c|c|c|c|c|c|c|c|}
\hline & \multicolumn{3}{|c|}{ Lenna } & \multicolumn{3}{c|}{ Mandrill } & \multicolumn{3}{c|}{ Lenna } & \multicolumn{3}{c|}{ Mandrill } \\
\hline Texture & 1 & 2 & 3 & 1 & 2 & 3 & 1 & 2 & 3 & 1 & 2 & 3 \\
\hline Mixed & 15.1 & 14.1 & 14.4 & 15.1 & 14.0 & 14.3 \\
\hline Latent & 21.9 & 29.5 & 26.8 & 22.5 & 26.5 & 21.6 \\
\hline Pattern & 20.6 & 31.2 & 26.0 & 21.7 & 27.2 & 21.4 & 14.2 & 14.4 & 15.1 & 14.1 & 14.3 \\
\hline & & 19.3 & 25.4 & 22.7 & 18.9 & 22.8 & 20.6 \\
\hline
\end{tabular}

pattern images so that they look like reflections dealt with in [2]. For blurring them, we use the Gaussian type point spread function with 3 (pixel) standard deviation. Additionally, in [2], we change the pixel-wise multiplicative model to the additive model used in our method.

In the RPCA (Fig. 4(b)), although we expect that the data matrix derived form a pattern image will be a low rank matrix, the latent image is also low rank, so the low frequency component was wrongly extracted when we tried to extract the pattern component ((b) top row). In the reflection removal (c), although we tried to extract the reflection component as the pattern, the low frequency component of the latent image was also extracted as a reflection component ((c) bottom row). On the other hand, our method can clearly separate the latent and the pattern image.

Quantitative evaluation based on PSNR: Table I shows the color PSNRs between separated results and their original images. Higher PSNR indicates higher similarity to the original image. The reconstruction degrees are different between cases when the pattern has continuous circularity or not at the boundary of an image. Therefore, both cases are, respectively, shown in the left and the right table. From the table, higher PSNRs are obtained when the pattern has the continuous circularity, especially in the Texture2 having the isotropic minute pattern. In contrast, the lowest PSNR is obtained in the Texture1 having flat regions. When a texture has flat regions, peak spectral values appear in the low frequency band. This makes it difficult to distinguish the peaks from the spectra of a latent image.

An experiment using practically scanned and shot images: Figure 5 shows results used actual scanned and shot images. The top row is the result of a scanned old image previously shown in Fig. 1. The bottom row is the result of a shot image taken from corridor to room inside through a blindfold sheet. In the scanned image, the image is successfully separated because the dimple pattern has evenness. In the shot image, the pattern cannot be completely removed, but can be reduced. This is caused by the opacity of the slit dots and the uneven sizes of the dots (sizes become small toward the bottom side).

Execution time: We implemented the aforementioned methods on a PC with Core i7 CPU@2.67GHz, RAM@24GB, and MATLAB2016b. The average execution time taken in one iteration of the ADMM used in the methods are, in our method: 0.80sec/Mpix, RPCA: $2.13 \mathrm{sec} / \mathrm{Mpix}$, and reflection removal: $16.34 \mathrm{sec} / \mathrm{Mpix}$ (author's code). Since the calculations 

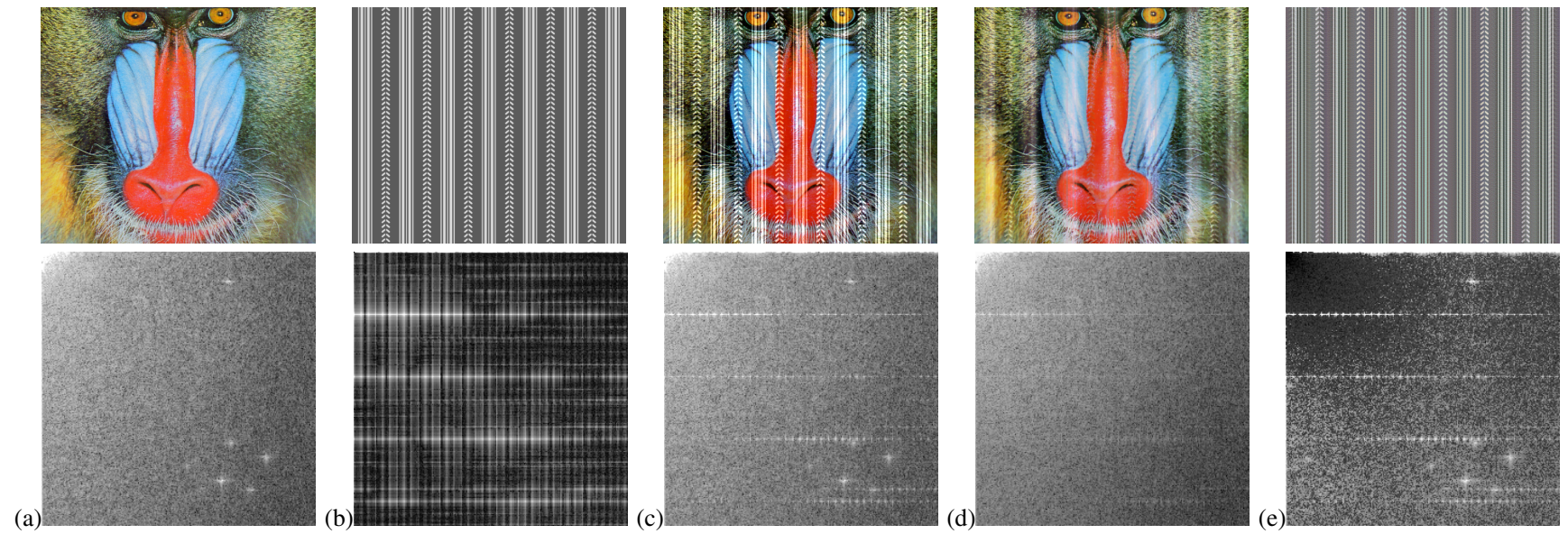

Fig. 3. Simulation results using an anisotropic non-circular pattern. The bottom images are their spectra. The intensity range of spectral images is normalized for displaying the details.

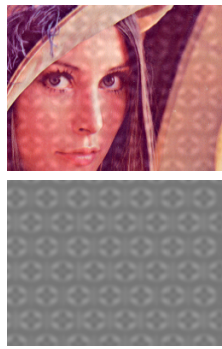

(a) Original

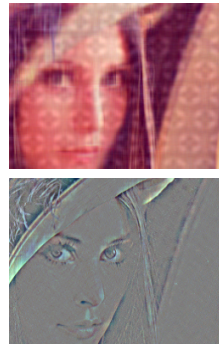

(b) RPCA [1]

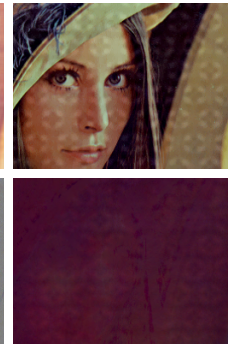

(c) Li et al. [2]

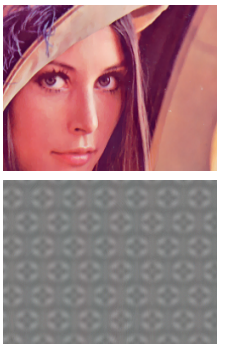

(d) Proposed

Fig. 4. Comparison with related methods. (a) is the observed image (top) and the ground truth pattern (bottom).

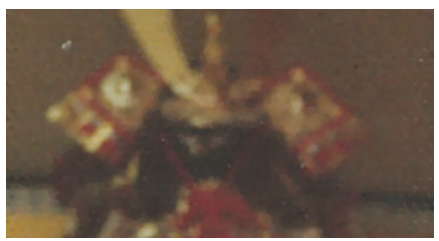

Latent

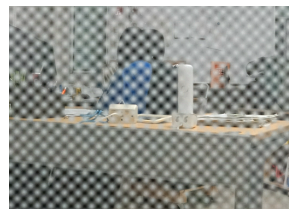

Original

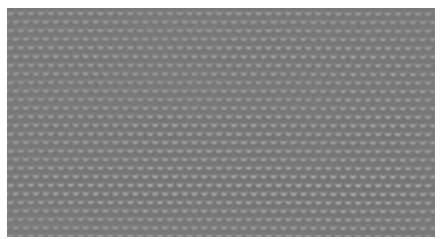

Pattern

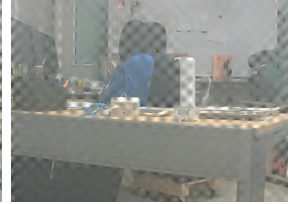

Latent

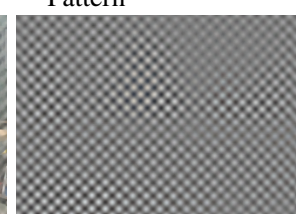

Pattern

Fig. 5. Results using an actual scanned and shot image.

used in our method are simpler than other methods, our method achieved fast execution time.

\section{CONCLUSION}

In this paper, we presented a method for removing a pattern artifact component from a degraded image. Since the amplitude spectra of the pattern image have sparsity, we characterize it as a mathematical optimization problem using a mixed norm consisting of the complex-valued norm and the $\ell_{1}$ norm. Although not all pattern noise can be removed by this method, we showed that it has better separation performance

compared to the conventional methods such as RPCA and reflection component removal.

Finally, we remark that the pattern noise removal problem considered would be related to cartoon-texture image decomposition methods that characterize texture by local lowrank regularization, e.g., [11], [12]. Such methods can handle pattern noise as texture, and thus might be effective for the problem. However, we also note that these methods require singular value decomposition at each iteration to solve the associated optimization problems, which means that they are much more computationally expensive than our method.

\section{REFERENCES}

[1] J. Wright, Y. Peng, Y. Ma, A. Ganesh, and S. Rao, "Robust principal component analysis: exact recovery of corrupted low-rank matrices by convex optimization," in Proc. NIPS, 2009, pp. 2080-2088.

[2] Y. Li and M. Brown, "Single image layer separation using relative smoothness," in Proc. IEEE CVPR, 2014, pp. 2752-2759.

[3] T.-S. T. Chan and Y. H. Yang, "Complex and quaternionic principal component pursuit and its application to audio separation," Signal Proce. Letters, vol. 23, no. 2, pp. 287-291, 2016

[4] S. Samarah, S. Obeidat, and R. Salman, "A Schur test for weighted mixed-norm spaces," Anal. Math., vol. 31, pp. 277-289, 2005.

[5] A. Benedek and R. Panzone, "The space $\ell_{p}$ with mixed norm," Duke Math. J., vol. 28, pp. 301-324, 1961

[6] M. Kowalski, "Sparse regression using mixed norms," Applied Comput. Harmonic Anal., vol. 27, no. 3, pp. 303-324, 2009.

[7] L. Rudin, S. Osher, and E. Fatemi, "Nonlinear total variation based noise removal algorithms," Physica D, vol. 60, no. 1-4, pp. 259-268, 1992.

[8] D. Gabay and B. Mercier, "A dual algorithm for the solution of nonlinear variational problems via finite elements approximations," Comput. Math. Apps., vol. 2, pp. 17-40, 1976.

[9] S. Boyd, N. Parikh, E. Chu, B. Peleato, and J. Eckstein, "Distributed optimization and statistical learning via the alternating direction method of multipliers," Founda. \& Trends in Machine Learn., vol. 3, no. 1, pp. 1-122, 2011.

[10] K. Shirai and M. Okuda, "Fft based solution for multivariable 12 equations using kkt system via fft and efficient pixel-wise inverse calculation," in Proc. IEEE ICASSP, 2014, pp. 2648-2652.

[11] H. Schaeffer and S. Osher, "A low patch-rank interpretation of texture," SIAM J. Imag. Sci., vol. 6, no. 1, pp. 226-262, 2013.

[12] S. Ono, T. Miyata, and I. Yamada, "Cartoon-texture image decomposition using blockwise low-rank texture characterization," IEEE T. Image Proce., vol. 23, no. 3, pp. 1128-1142, 2014. 